\title{
Lower urinary tract symptoms in women with benign joint hypermobility syndrome: a case-control study
}

\author{
H. Mastoroudes • I. Giarenis • L. Cardozo • \\ S. Srikrishna • M. Vella • D. Robinson • H. Kazkaz • \\ R. Grahame
}

Received: 24 October 2012 / Accepted: 30 January 2013 /Published online: 23 February 2013

(C) The International Urogynecological Association 2013

\begin{abstract}
Introduction and hypothesis Benign joint hypermobility syndrome (BJHS) is a connective tissue disorder associated with joint hypermobility. BJHS is under-recognised by medical professionals and is poorly managed. The aim of our study was to determine whether lower urinary tract symptoms (LUTS), including urinary incontinence (UI) and anterior compartment prolapse, are more common in women with BJHS than in the normal population.
\end{abstract}

H. Mastoroudes $\cdot$ I. Giarenis $\cdot$ L. Cardozo $\cdot$ S. Srikrishna $\cdot$

M. Vella $\cdot$ D. Robinson

King's College Hospital NHS Foundation Trust, London, UK

I. Giarenis

e-mail: Ilias.giarenis@nhs.net

L. Cardozo

e-mail: linda@lindacardozo.co.uk

S. Srikrishna

e-mail: Sushmasrikrishna@nhs.net

M. Vella

e-mail: Maria.vella@nhs.net

D. Robinson

e-mail: Dudley.robinson@nhs.net

H. Kazkaz $\cdot$ R. Grahame

University College Hospital NHS Foundation Trust,

Denmark Hill, UK

H. Kazkaz

e-mail: hanadikazkaz@hotmail.com

R. Grahame

e-mail: rodneygrahame@aol.com

H. Mastoroudes $(\square)$

Department of Urogynaecology, King's College Hospital,

Suite 8, 3rd floor Golden Jubilee Wing,

Denmark Hill SE5 9RS, UK

e-mail: heleni.mastoroudes@nhs.net
Methods This was a prospective case-control study. Women diagnosed with BJHS according to the Brighton criteria were recruited from a tertiary referral clinic. Controls were recruited from hospital personnel. Both groups completed the King's Health Questionnaire (KHQ) and the Prolapse Quality of Life Questionnaire (P-QoL). Objective assessment of pelvic organ prolapse (POP) was undertaken using the Pelvic Organ Prolapse Quantification (POP-Q) system. Analyses were performed using SPSS version 17.0. The statistical difference was analysed using McNemar's test. Comparison of QoL scores was performed with the Wilcoxon signed-rank test.

Results Sixty individuals were recruited and matched with 60 healthy women. The prevalence of UI in those with BJHS was significantly higher than in controls $(73.3 \%$ vs. $48.3 \%$ ). The impact of UI on QoL was statistically significant in all domains of the KHQ. There was a significant difference between groups in most urinary-specific symptoms of the KHQ. A significant number of women with BJHS suffer from voiding difficulties. Prolapse of the anterior vaginal wall was objectively more severe in those with BJHS.

Conclusions Women with BJHS have LUTS and anterior compartment prolapse, which significantly impair their QoL. It is important to identify women who are symptomatic. The addition of a systematic active case-finding approach may be more effective in identifying these cases.

Keywords Benign joint hypermobility syndrome $\cdot$ Lower urinary tract symptoms $\cdot$ Quality of life

\section{Introduction}

Urinary incontinence (UI) is a common condition that significantly impairs quality of life (QoL) [1]. It is defined by 
the International Continence Society (ICS) and International Urogynaecology Association (IUGA) as the involuntary loss of urine [2]. The prevalence ranges from $12.8 \%$ to $46 \%$ [3]. Definitions of lower urinary tract function and dysfunction have also been standardised as a consensus document between IUGA/ICS [2]. Urinary incontinence is multifactorial and increases with age [4]. Age-related urogenital atrophy with its associated reduction in skin collagen content is assumed to be a causative factor. Inherited, intrinsic genetic abnormalities of collagen synthesis may also contribute to UI. These may range from more severe forms of connective tissue disorders, such as Marfan's syndrome and type IV Ehlers-Danlos syndrome, to benign joint hypermobility syndrome (BJHS), also known as the hypermobile type of Ehlers-Danlos (or Ehlers-Danlos type III) syndrome. The basic pathophysiology of BJHS is due to an underlying abnormality in collagen where the ratio of type III to type I collagen is increased [5]. This means that tissue is more stretchable, which may result in generalised tissue damage. BJHS usually presents with a variety of musculoskeletal problems ranging from joint subluxation to tendosynovitis. It may also affect multiple other systems, including the musculotendinous pelvic floor. This can result in pelvic floor problems in women, such as pelvic organ prolapse (POP) and UI. Given the background abnormality of collagen in BJHS, the aim of this case-control study was to identify whether the prevalence of lower urinary tract symptoms (LUTS), including UI, in the BJHS population is greater than in normal women. The relationship between BJHS, voiding difficulties and prolapse was also explored.

\section{Materials and methods}

All patients referred to the tertiary referral hypermobility clinic at University College Hospital with a diagnosis of BJHS were invited to participate in this study between October 2010 and August 2011. University College Hospital has a multinational catchment population and is also one of the three adult hypermobility clinics in the UK. Patients had been sent information leaflets regarding this study with their appointment letters. All control individuals were recruited from King's College Hospital and included general gynaecology patients, medical personnel and other hospital staff. Ethical approval was obtained from the local ethics committee, and all women gave informed consent prior to participation. Inclusion criteria were women aged between 18 and 60 years who understood English. Those who had previous pelvic floor surgery were excluded. All study group participants recruited from University College Hospital were given a diagnosis of BJHS having fulfilled the Brighton criteria [6]. All control participants were screened for BJHS and excluded from participation if they fulfilled the same criteria.

The Brighton criteria ("Appendix 1") comprise sets of major and minor criteria. A diagnosis of BJHS is made when either two major, one major and two minor or four minor criteria were satisfied [7]. The Beighton score ("Appendix 2"), which is one of the major criteria, was once considered the gold standard for recognising hypermobility [6].

Patients were matched according to age \pm 2 years, exact parity and ethnicity. The recruitment of controls was performed following the recruitment of cases. All participants were asked to complete the validated King's Health Questionnaire (KHQ) and the Prolapse Quality Of Life (P-QOL) questionnaire. The KHQ is a disease-specific validated $\mathrm{QoL}$ questionnaire used in the assessment of women with UI [1]. It evaluates nine QoL domains and nine LUTS-specific areas. The QoL domains include general health perception (GHP), incontinence impact (II), role limitations (RL), physical limitations (PL), social limitations (SL), personal limitations (PL), effect on emotions, effect on sleep and energy and severity. Each domain is given a calculated score out of 100 . The higher the score, the more significant the negative impact on QoL. LUTS include frequency, nocturia, urgency, urgency incontinence (UUI), stress incontinence (SUI), nocturnal enuresis, intercourse incontinence, urinary tract infections (UTI) and bladder pain. Each symptom is graded as mild, moderate or severe if present. Questionnaire respondents are given the option of not ticking a box if they feel they do not have a particular symptom listed on the questionnaire. Prevalence is calculated according to whether symptoms are present or not.

The P-QoL is a disease-specific, validated questionnaire developed to assess QoL in women with urogenital prolapse [8]. It focuses on the same nine domains used in the KHQ but in relation to prolapse. It also has a section for urinary-, bowel- and prolapse-specific symptoms related to prolapse. We only included urinary symptoms in relation to voiding dysfunction, such as straining, stream quality, incomplete emptying and postmicturition dribbling. Each symptom is graded according to severity, and their prevalence is calculated according to whether symptoms were present or not, as with the KHQ. Prolapse was measured to examine how it related to voiding difficulties. Objective assessment of prolapse was made with POPstix ${ }^{\circledR}$ (a wooden measuring spatula with $1-\mathrm{cm}$ intervals) using the International Continence Society (ICS) Pelvic Organ Prolapse Quantification (POP-Q) system [9]. This system is a standardised and descriptive method that allows measurement of the support of nine specific points of the vagina in centimetres. We then compared KHQ scores, voiding dysfunction symptoms from the P-QoL 
and all nine points of the POP-Q between study patients and controls. The power calculation was based on the prevalence of UI in women with BJHS in a study by Jha et al. [10]. The prevalence of incontinence in this study was $60 \%$ in the BJHS group, and it was calculated that 60 participants in each group would result in a power of $80 \%$ to detect a difference of $30 \%$ at a $5 \%$ significance level.

Statistical methods

All analyses were performed using SPSS version 17.0 (SPSS Inc., Chicago, IL., USA). Statistical difference for categorical variables in case of $2 \times 2$ tables were analysed using McNemar's test. Data analysis comparing QoL scores was performed with the Wilcoxon signed-rank test. Paired $t$ test was used to compare POP-Q measurements. The Kendall-Tau $b$ test was used to measure the association between two measured quantities. Statistically significant difference was $p<0.05$.

\section{Results}

One hundred and twenty individuals (60 study patients, 60 controls) were recruited during a 10-month period. All patients were matched with healthy volunteers according to age $(p=0.096)$, parity $(p=0.321)$ and ethnicity ( $p=$ 0.188 ). The age range was from 18 to 60 (mean 39.4) years. Median parity was 1 (range $0-3$ ). The prevalence of UI, as calculated from the KHQ, in those with BJHS was $73.3 \%$ $(44 / 60)$ and $48.3 \%(29 / 60)(p=0.009)$ in controls. Sixty-two per cent $(37 / 60)$ of the study group had UUI, which was significantly more than the $38.3 \%(23 / 60)$ in the control group $(p=0.017)$. Although the prevalence of SUI in the study group was $63.3 \%(38 / 60)$ compared with $36.7 \%$ in the control group, this was not statistically significant $(p=$ 0.141 ). There was a significant difference between study and control groups in all the other urinary-specific symptoms of the KHQ except for intercourse incontinence (Fig. 1).

Symptoms of voiding dysfunction are not incorporated in the symptom-specific section of the KHQ. These results were obtained from the P-QoL (Table 1). It is evident that a significant number of women with BJHS suffer from voiding dysfunction. Most patients in the study group $(63.3 \%)$ felt they had incompletely emptied their bladders in comparison with a small number $(23.3 \%)$ in the control group. A considerable proportion (48.3\%) of women with BJHS felt they needed to strain in order to empty their bladders. Only $13.3 \%$ of the control group felt they needed to strain in order to empty their bladders. This may have led to the high percentage of women with BJHS suffering from UTI and bladder pain (Fig. 1).

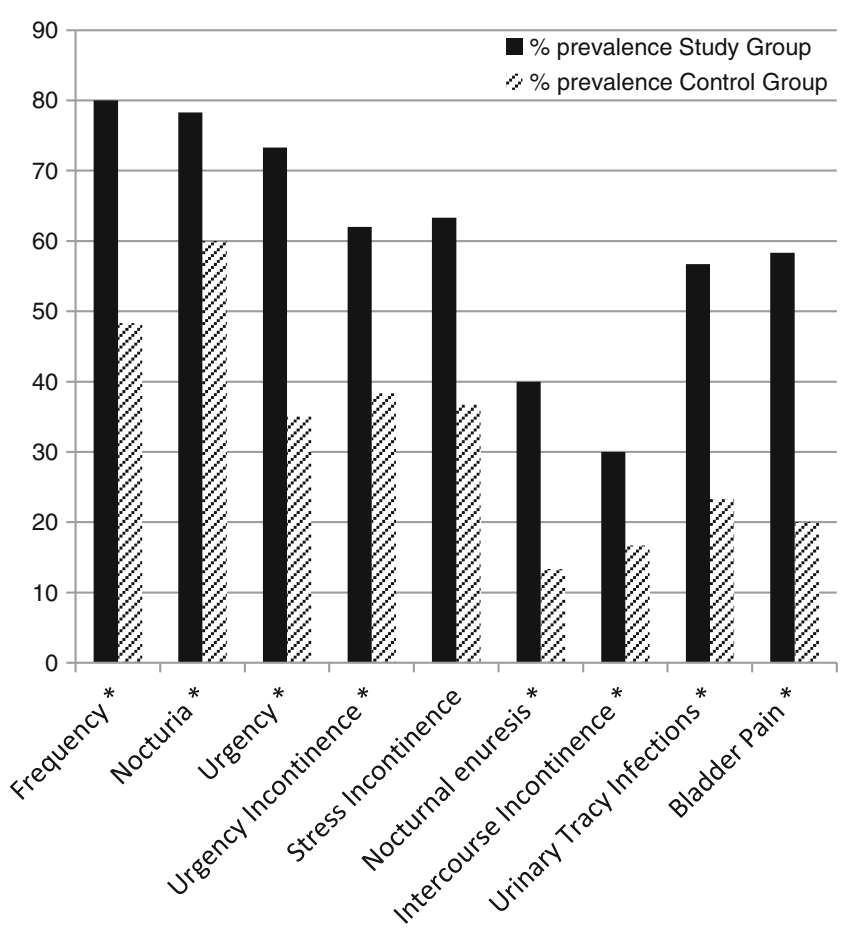

Fig. 1 Comparison of prevalence (\%) of the individual King's Health Questionnaire (KHQ) urinary-symptom-specific questions between study and control groups. $* P<0.05$

The impact of UI on QoL between cases and controls was statistically significant in all nine domains of the KHQ (Fig. 2).

When considering prolapse of the anterior compartment, there was a significant difference in prolapse between groups (Table 2). There was also a significant correlation between prolapse in the anterior compartment and incomplete bladder emptying $(p=0.037)$.

\section{Discussion}

This is the largest case-control study to our knowledge comparing the prevalence of UI in those with BJHS to a control group. As expected, the prevalence of UI was significantly greater in the hypermobile population. When patients were asked about the severity of incontinence, it

Table 1 Results of Prolapse Quality of Life (P-QOL) urinary voiding difficulty questions

\begin{tabular}{llcc}
\hline Urinary symptoms P-QOL & Study group & Control group & $P$ value \\
\hline Straining & $48.3 \%(29 / 60)$ & $13.3 \%(8 / 60)$ & $<0.001$ \\
Poor stream & $38.3 \%(23 / 60)$ & $8.3 \%(5 / 60)$ & $<0.001$ \\
Incomplete bladder & $63.3 \%(38 / 60)$ & $23.3 \%(14 / 60)$ & $<0.001$ \\
$\begin{array}{l}\text { emptying } \\
\text { Postmicturition dribble }\end{array}$ & $46.7 \%(28 / 60)$ & $30 \%(18 / 60)$ & 0.091 \\
\hline
\end{tabular}


Fig. 2 Comparison of the mean King's Health Questionnaire (KHQ) scores on the $y$ axis between study and control groups. (The maximum score of 100 was not incorporated in the $y$ axis to allow easier data interpretation). All domains were statistically significant; $p<0.05$

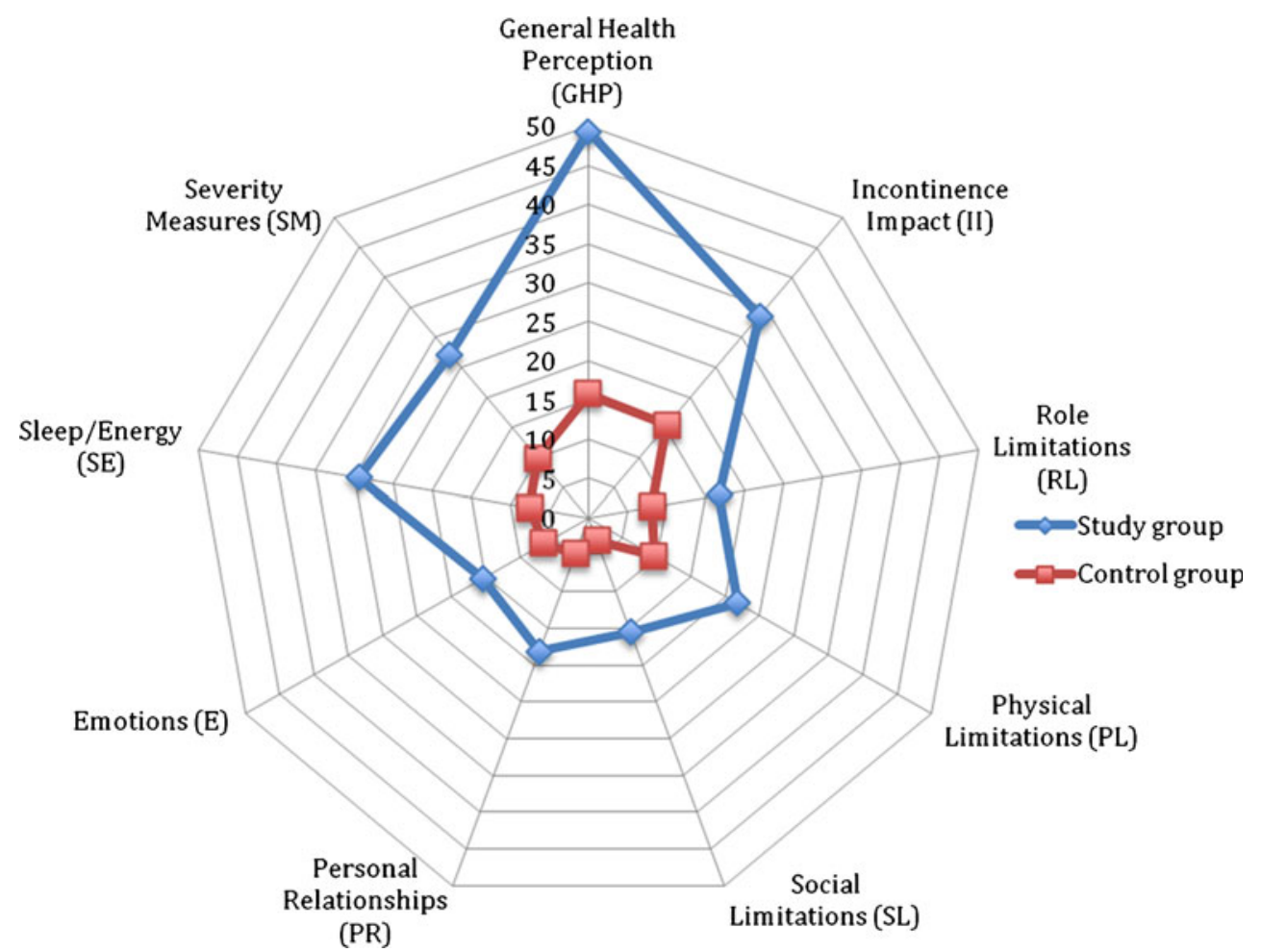

was mostly mild on a severity scale. Interestingly, a considerable number of women in the study group also suffered from voiding dysfunction. UI had a considerably negative impact on all aspects of QoL. It is possible that UI has an impact on the mechanical aspects of their BJHS, which affects all domains of QoL, resulting in a significant difference. Women with BJHS have a wide variety of problems in relation to the lower urinary tract and lower bowel. The literature indicates that these patients experience UI and anal incontinence and also obstructed defecation.

Table 2 Results of the mean Pelvic Organ Prolapse Questionnaire (POP-Q) system measurements

\begin{tabular}{lllc}
\hline POP-Q points & $\begin{array}{l}\text { Study group } \\
\text { (SD) }\end{array}$ & $\begin{array}{l}\text { Control group } \\
(\mathrm{SD})\end{array}$ & $P$ value \\
\hline $\mathrm{Aa}$ & $-0.4(1.6)$ & $-1.8(1.4)$ & $<0.001$ \\
$\mathrm{Ba}$ & $-0.3(1.5)$ & $-1.5(1.4)$ & $<0.001$ \\
$\mathrm{Ap}$ & $-1.3(1.5)$ & $-2.3(1.1)$ & $<0.001$ \\
$\mathrm{Bp}$ & $-1.2(1.5)$ & $-2.3(1.1)$ & $<0.001$ \\
$\mathrm{C}$ & $-7.1(1.5)$ & $-7.6(1.4)$ & 0.027 \\
D & $-8.6(1.3)$ & $-8.3(2.5)$ & 0.67 \\
Genital hiatus (GH) cm & $3.1(0.8)$ & $2.9(0.8)$ & 0.177 \\
Perineal body (PB) cm & $3.0(0.7)$ & $3.5(0.8)$ & $<0.001$ \\
Total vaginal length & $8.9(0.9)$ & $8.9(0.8)$ & 0.799 \\
$\quad$ TVL) cm & & & \\
\hline
\end{tabular}

$S D$ standard deviation
The prevalence of incontinence ranges from $42 \%$ to $60 \%$ [10]. This has been confirmed by our study, albeit with a greater prevalence of $73 \%$. This may be due to our larger cohort. Although the reported incidence of SUI and UUI is similar, only the difference in UUI between study and control groups was statistically significant. A significant portion of women with BJHS felt their LUTS had a negative impact on QoL. This is contrary to the published data from Jha et al. [10], which showed that despite a statistically significant proportion of women with UUI and anal incontinence, their impact on QoL was minimal. When considering the second domain (incontinence impact on QoL) of the KHQ in relation to those with UI, a significant portion felt that this affected them moderately to severely. Enquiring about and identifying these symptoms can lead to the provision of numerous treatment options that can result in a considerable improvement in QoL.

It has been postulated [11] that women with BJHS have a higher incidence of obstructed defecation due to either pelvic floor weakness or a connective tissue disorder inhibiting appropriate bowel-wall motility. This may also be true with regard to voiding dysfunction, which we have shown to be problematic in the hypermobile cohort. This may again be either due to a connective tissue defect in the pelvic floor, resulting in a cystocele and therefore an anatomical problem, or it may be functional. These could also be due to a defect in the connective tissue in the bladder wall affecting bladder 
contractility and causing voiding dysfunction secondary to detrusor failure. It is also important to consider that LUTS maybe the presenting complaint of patients who may not perceive that their BJHS is significant or a variant of normal.

We acknowledge that our study had some weaknesses. Although incontinence was identified by means of questionnaires, this does not always necessarily correlate with the urodynamic diagnosis. The bladder has been described as an "unreliable witness", and urodynamic investigation would be ideal in order to identify types of incontinence [12]. This would be interesting, as theoretically, the pathophysiology underlying UI in those with BJHS is more likely to be due to a weakness in the pelvic floor or intrinsic sphincter deficiency rather than detrusor overactivity. In addition, QoL questions on both the KHQ and P-QoL are validated; the sections on LUTS in relation to incontinence and prolapse provide only a subjective assessment. They would not be considered reliable for objectively assessing the lower urinary tract. Whilst the researcher collecting the data did ask study participants whether they would consider further investigation/therapy for their incontinence, it would have been useful to use a more structured survey questionnaire or patient symptom perception questionnaire to establish the exact proportion.

\section{Conclusion}

Incontinence is a source of much embarrassment to women. However, clinicians rarely acknowledge the substantial impact it has on the QoL of women with BJHS, as a large and understandable proportion of time is spent in specialised rheumatology/hypermobility clinics considering all other aspects of this condition. Thus an insignificant amount of time, or sometimes none at all, is spent asking about gynaecological issues. Underdiagnosis of these issues may be in part due to patient underreporting of their symptoms to their physicians and in part to a failure by general practitioners and rheumatologists to routinely screen for these symptoms. It is important to identify women who are symptomatic. The addition of a systematic, active case-finding approach may be more effective in identifying these cases. Such a high prevalence of incontinence may justify the need for an integrated continence pathway in the larger specialised hypermobility units in the country.

Acknowledgements We thank Monika Vij for protocol design. King's College Hospital NHS Foundation Trust funded research and development grant.

Conflicts of interest None.

\section{Appendix 1}

Table 3 Brighton criteria

Criteria

Major

1. Beighton score of 4-9 or greater currently or historically

2. Arthralgia for longer than 3 months in four or more joints

Minor

1. Beighton score of 1,2 or $3-9(0,1,2$, or 3 if aged $50+$ years)

2. Arthralgia ( $>3$ months) in one to three joints, or back pain (> 3 months), spondylosis, spondylolysis/spondylolisthesis

3. Dislocation/subluxation in more than one joint or in one joint on more than one occasion

4. Soft-tissue rheumatism; three or more lesions ( e.g. epicondylitis, tenosynovitis, bursitis)

5. Marfanoid habitus

6. Abnormal skin: striae, hyperextensibilty, thin skin, papyraceous scarring

7. Eye signs: drooping eyelids or myopia or antimongoloid slant

8. Varicose veins or uterine/rectal prolapse

\section{Appendix 2}

Table 4 Nine-point Beighton hypermobility score

\begin{tabular}{lll}
\hline & Right & Left \\
\hline $\begin{array}{l}\text { Passively dorsiflex the 5th metacarpophalangeal } \\
\text { joint to }>90\end{array}$ & 1 & 1 \\
Oppose the thumb to the volar aspect of the & 1 & 1 \\
ipsilateral forearm & 1 & 1 \\
Hyperextend the elbow to $>10$ & 1 & 1 \\
Hyperextend the knees to $>10$ & 1 & \\
Place hands flat on the floor without bending knees & 9 & \\
Maximum total & \\
A score of $>4-9$ is considered to indicated BJHS, \\
although there is no universal agreement on the \\
accurate value.
\end{tabular}

$B J H S$ benign joint hypermobility syndrome

\section{References}

1. Kelleher CJ, Cardozo LD, Khullar V, Salvatore S (1997) A new questionnaire to assess the quality of life of urinary incontinent women. Br J Obstet Gynaecol 104:1374-1379

2. Haylen BT, de Ridder D, Freeman RM et al (2010) An International Urogynaecological association (IUGA)/ International Continence Society (ICS) joint report on the terminology for female pelvic floor dysfunction. Int Urogynecol J 21:5-26

3. Botlero R, Urguhart DM, Davis SR, Bell RJ (2008) Prevalence and incidence of urinary incontinence in women: review of the 
literature and investigation of methodological issues. Int J Urol 15(3):230-4

4. Hannestad YS, Rortveit G, Sandvik H et al (2000) A community based epidemiological survey of female urinary incontinence: The Norwegian EPICONT Study. J Clin Epidemiol 53:1150-1157

5. Child AH (1986) Joint hypermobility syndrome: Inherited disorder of collagen synthesis. J Rheumatol 13:239-46

6. Beighton P, Grahame R, Bird H (1999) Hypermobility of joints, 3rd edn. Springer-Verlag, London

7. Graham R, Bird H (2001) British consultant rheumatologists' perception about the hypermobility syndrome; A national survey. Rheumatology 40:559-62

8. Digesu A, Khullar V, Cardozo L, Robinson D, Salvatotre S (2005) P-QOL: a validated questionnaire to assess the symptoms and quality of life of women with urogenital prolapse. Int Urogynecol J $16: 171-181$

9. Bump RC, Mattiasson A, Bo K, Brubaker LP, DeLancey JO, Klarskov $P$ et al (1996) The standardisation of terminology of female pelvic organ prolapse and pelvic floor dysfunction. Am J Obstet Gynecol 175(1):10-17

10. Jha S, Arunkalaivanan R, Situayake D (2007) Prevalence of incontinence in women with benign joint hypermobility syndrome. Int Urogynecol J 18:61-64

11. Manning J, Korda A, Bennes C, Solomon M (2003) The association of obstructive defaecation, lower urinary tract dysfunction and the benign joint hypermobility syndrome: A case control study. Int Urogynaecol J 14:128-132

12. Hashim H, Abrams $P$ (2006) Is the bladder a reliable witness for predicting detrusor overactivity? J Urol 175:191-195 